\title{
Electromagnetic Equivalent Models for Printed Circuit Boards inside a Metallic Enclosure Using a Coaxial-to-Waveguide Transition Calibration
}

\author{
Antonio José Lozano-Guerrero, Juan Monzó-Cabrera, Member IEEE, Francisco Javier Clemente- \\ Fernández, José Fayos-Fernández, Juan Luis Pedreño-Molina, and Alejandro Díaz-Morcillo, Senior \\ Member IEEE
}

\begin{abstract}
Equivalent models of printed circuit boards are useful to simplify electromagnetic problems by reducing the computational costs of numerical simulations. In this paper, a new procedure for obtaining a simple equivalent model of printed circuit boards inside metallic enclosures is presented. The equivalent model is obtained in two steps: first we precisely characterize the coaxial to waveguide transitions used during measurements by means of an inverse procedure and then during a second inverse procedure, we carry out simulations by concatenating these transitions and the equivalent model and comparing to measurements. The optimized parameters for the equivalent model are: thickness, dielectric constant and the electric conductivity. Results for a printed circuit board in three scenarios have been obtained by using a sweep and two different optimization techniques. Benefits and drawbacks of the model are discussed. Results indicate that this procedure produces very precise characterization of the equivalent model of PCBs depending on the position and orientation of this device within the enclosure.
\end{abstract}

Index Terms - Calibration, cascade systems, modeling, waveguide transitions, shielding.

\section{INTRODUCTION}

$\mathrm{T}$ HE electrical behaviour of printed circuit boards (PCBs) inside of a protecting cabinet is a key issue to understand the shielding performance of an enclosure with built-in apertures designed for airing purposes. On the one hand, the components on a PCB have to be protected against radiated electromagnetic (EM) interferences that may lead to an unwanted or uncontrolled behaviour of the device. On the other hand, the presence of the PCB within the protecting

Manuscript received October 26, 2011; accepted December 21, 2011. This work was supported in part by the Fundación Séneca, Agencia de Ciencia y Tecnología de la Región de Murcia, under the Séneca predoctoral fellowship with reference $16381 / \mathrm{FPI} / 10$ and under the project $00700 / \mathrm{PPC} / 04$

A. J. Lozano-Guerrero, J. Monzó-Cabrera , F. J. Clemente-Fernández, J. Fayos-Fernández, J.L. Pedreño-Molina, and A. Díaz-Morcillo, are with the Departmento de Tecnologías de la Información y las Comunicaciones of Universidad Politécnica de Cartagena, Cartagena, E-30202, Spain (phone; +34968326468 fax: +34968325973 e-mail: antonio.lozano@upct.es). cabinet forces a redistribution of the field patterns inside the enclosure shifting the original problem when the case was empty to a different one.

The EM interactions with PCBs have been studied in several works. The simplest approximation to model a PCB in a shielding scenario consists of modelling the tracks and the composite structure of the board with the components attached to it as a perfect electric conductor (PEC) plate with the same dimensions as the PCB. Such approximation assumes a homogeneous ground plane as the main contribution for the model. This simply model has been used in studies with semianalytical [1] and numerical [2] methods. However, this model does not take into account the losses due to the circuitry on the $\mathrm{PCB}$, neither the ones due to all the materials building up the device.

In [3] it was stated that a populated PCB could be modelled for computational purposes by a homogeneous sheet of a lossy conductor and characterized by its reflection and transmission coefficients, depending on the requirements of the computational algorithm. An accurate EM equivalent model of a PCB without a ground plane was modelled by a slab of homogeneous dielectric material that filled up the crosssection of the waveguide in [4]. The perturbation caused by several types of grounded and floating PCBs within an enclosure was empirically studied in [5] and compared with computational simulations by using the TLM technique. The study concluded that a reasonable equivalent model could be a sheet of a conducting plane plus a lossy dielectric with the same dimensions as the commercial PCB. Nevertheless, the experimental characterization of such work introduced setup mismatches into the PCB characterization, so the coupling effects where not taken into account.

This work goes a step further in such reasonable approximation by applying the experimental technique used in [6] for providing accurate results. The damping effect produced by the lossy materials of any PCB can be reproduced computationally by adding a lossy layer to the conducting plane of its EM equivalent model. This model assumes that both the PEC plate and the lossy layer have the same surface area than the original $\mathrm{PCB}$, but it requires the characterization of the thickness $(d)$, dielectric constant $\left(\varepsilon_{r}\right)$ and electrical $\operatorname{conductivity}(\sigma)$ of the lossy slab (see Fig. 1). 
Traditionally, measurements performed in protecting cabinets to assess their shielding features are carried out by using coaxial probes that are inserted into the metallic cavity. These coaxial probes attached to the metallic enclosure are often referred as coaxial-to-waveguide transitions in microwave bibliography.

In [7] an efficient analytical tool was presented to evaluate the EM coupling between two wires within an empty metallic cavity. Nevertheless, some issues may affect the accuracy of the results and distort the assessment, such as the mechanical tolerances in manufacturing the enclosure, the soldering quality of the ground line of the coaxial cables or even the inclusion of connectors and/or adaptors. When evaluating the EM perturbation inside an enclosure when it is loaded with a $\mathrm{PCB}$, those issues must be handled properly, otherwise their influence might be erroneously attributed to the PCB perturbation under study, and consequently, the calculation of the EM equivalent model parameters would be affected as shown in [5].

During this work the method presented in [8] is used to provide accurate results for the PCB equivalent model inside the cavity. However, in this case we go a step further to overcome any other external factors such as the coupling of coaxial probes used in measurements. The method is based on the accurate experimental characterization of the $S$-parameters of both coaxial probes installed in the enclosure by using the empirical data registered from a rigorous calibration procedure. Once the probes are characterized, their scattering matrices are used during the needed simulations of the inverse procedure to obtain the PCB equivalent model.

In [9] a new proposed definition for the shielding effectiveness measurement of enclosures would require a set of different representative contents (RCs) for loading the protecting cabinets under test. The method presented in this article can be used when an accurate model for a representative content is required.

\section{THEORY}

Fig. 2 shows the scheme of the new two-step procedure used in this work to obtain the PCB equivalent model. During the first step, the coaxial to waveguide transitions used in the experimental measurements are fully characterized and their complex scattering matrices are obtained for all the frequency points under study. The details of this calibration procedure are explained in subsection $\mathrm{A}$.

The second step obtains the PCB equivalent model. With that purpose, the scattering matrices of these transitions are used to simulate the whole enclosure by concatenating them to the full wave simulation of the waveguide thru that contains the PCB equivalent model. This simulation is then compared to measurement and the PCB model parameters properly optimized to match measurements. A detailed description of this second step is provided in subsection B.

Once the transitions have been properly characterized the simulation time is not only reduced by the fact that there is a reduction of the computational domain but also from changing from a resonant problem (enclosure) to a transmission one (waveguide line). This last issue may be very interesting in time-domain simulations where the convergence is slower and especially when using global optimizing procedures where the number of evaluations of the fitting function is very high.

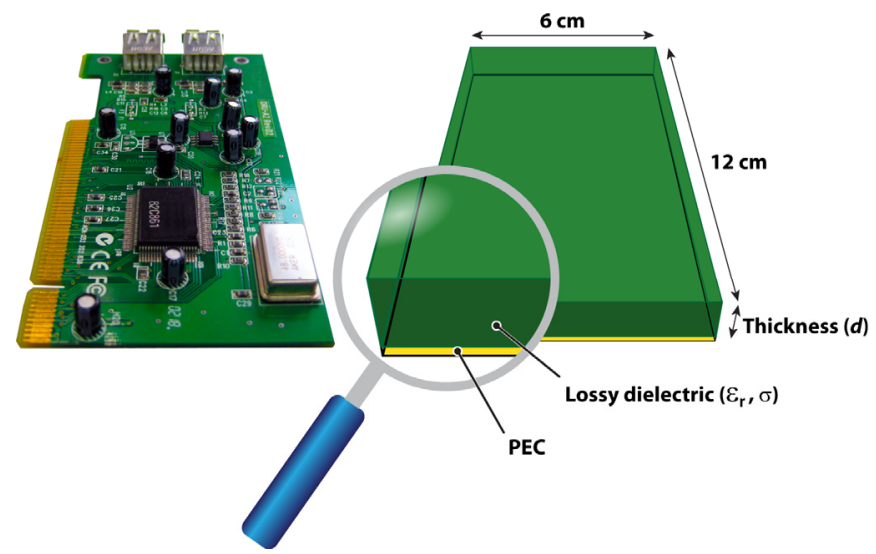

Fig. 1. PCB equivalent model used in this work.

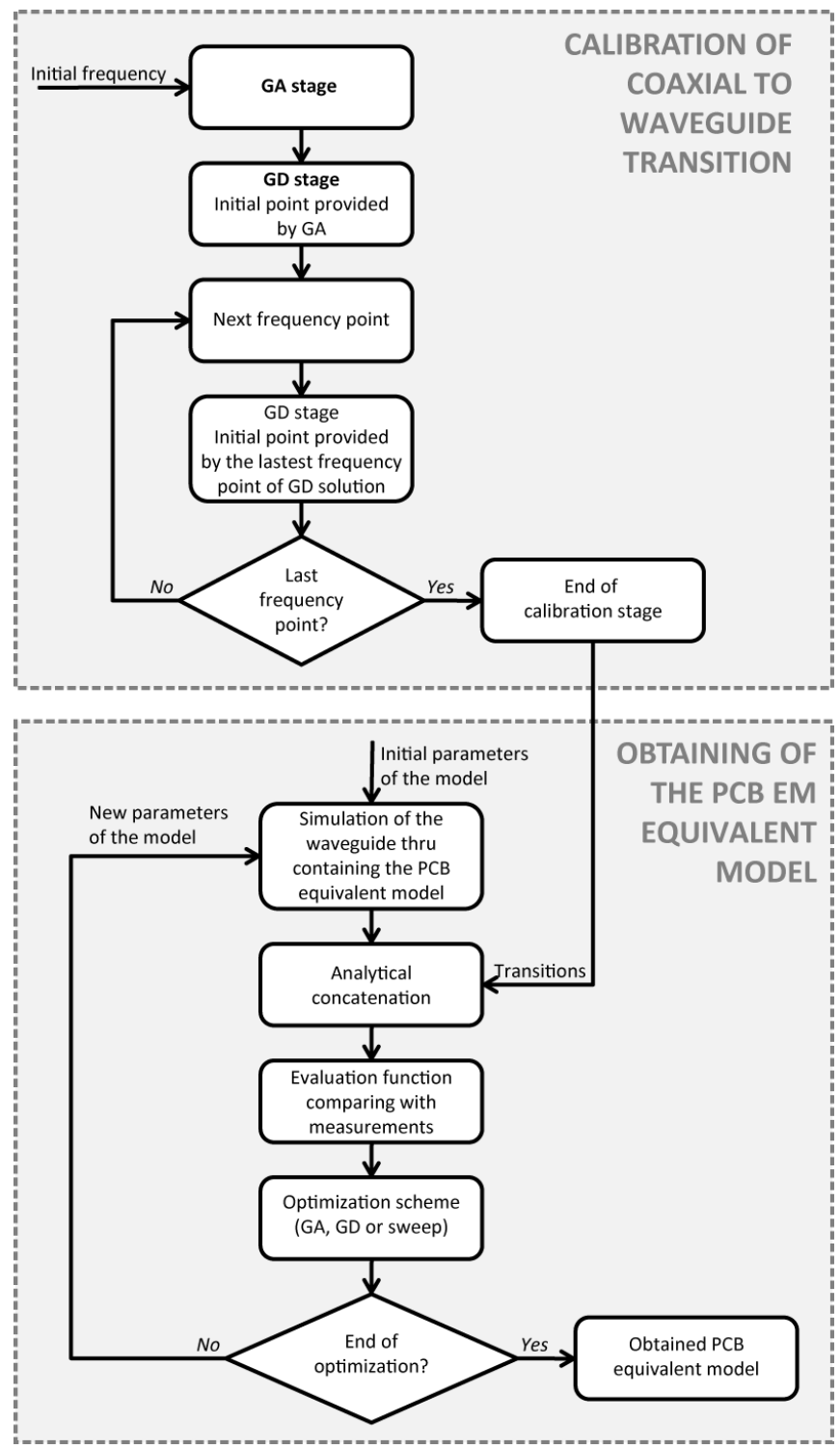

Fig. 2. Scheme of the two-step procedure for obtaining the PCB equivalent model. 


\section{A. Inverse calibration procedure for the coaxial-to-waveguide transitions of the metallic enclosure}

Coaxial monopoles within metallic cavities can also be studied as coaxial-to-waveguide transitions. A previous study for deembedding and unterminating coaxial-to-waveguide transitions was presented in [6]. In that work, experimental measurements and analytical calculations were combined with a genetic algorithm (GA) and gradient descent (GD) based technique to precisely obtain the frequency-dependent complex scattering matrix of each transition. The way to characterize these transitions is to reduce the error when comparing the measured standards (thrus and/or shorts) to analytical simulations where the unknown variables are the scattering parameters of the transitions [6]. The three cavities method described in [10] for coaxial probe modeling has also been used in this work for validating purposes.

The procedures described in [6] and [10] have been slightly modified here in order to be able to work with a complete metallic cavity instead of a cavity built up of different assembled parts. In fact in this work, the enclosure was virtually divided into three parts as shown in Fig. 3: two coaxial-to-waveguide transitions and a central waveguide part. Each of these virtual parts is characterized by their scattering matrix and then concatenated to obtain the whole response of the enclosure.

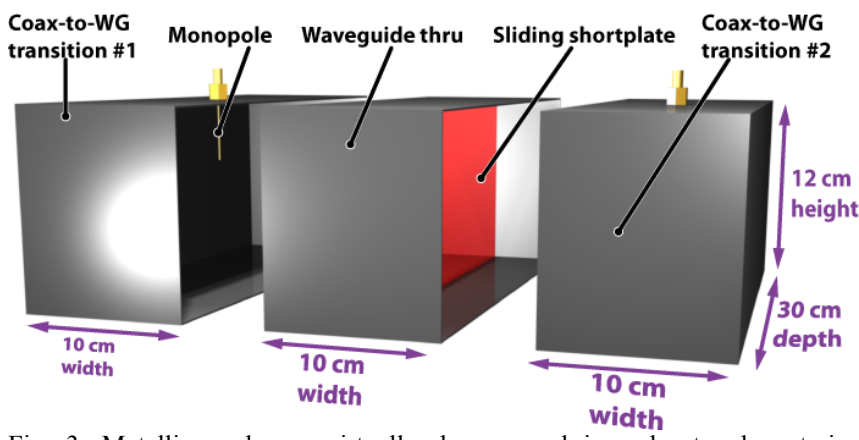

Fig. 3. Metallic enclosure virtually decomposed in order to characterize coaxial-to-waveguide transitions.

The calibration measurements for the reflection behavior of each transition were performed by positioning a sliding shortplate at $1.25,5$ and $8.75 \mathrm{~cm}$ away from the reference plane of the virtual waveguide port of transition 1 . The measurement of the whole empty enclosure, which implies the virtual $10 \mathrm{~cm}$ waveguide in between both transitions, was performed to define the thru behavior. The implemented method assumed a $\mathrm{TE}_{10}$ propagation mode as explained in [6]. In order to reduce computing times during the transitions calibration stage, the results of a genetic algorithm were used for seeding a gradient based algorithm [6] as shown in Fig. 2. The genetic algorithm was used for finding the global minimum error between measured and simulated data at the first frequency point with 100 individuals and 200 generations. The mutation function adds a random number taken from a Gaussian distribution with mean 0 to each entry of the parent vector. The algorithm shrinks the variance in each coordinate linearly until it reaches 0 at the last generation. Afterwards, the obtained result was introduced as the initial searching point in the gradient based method to refine the solution of the first frequency point. For the subsequent frequency points GA are not longer used and the solution obtained in the previous frequency point with GD is used as the initial guess for the GD at the next frequency.

The error evaluation function used in this work takes into account the magnitude of the difference between the measured complex scattering parameters $\left(S_{i j}^{m}\right)$ and the simulated ones $\left(S_{i j}^{s}\right)$ evaluated for each calibration setup (reflection and transmission). The expression used to evaluate the error at each frequency point when short-circuiting the transition is shown in equation (1) whereas for the transmission configuration (thru) equation (2) is applied [6].

$$
\begin{gathered}
f_{\text {short }}=\left|S_{n n}^{m}-S_{n n}^{s}\right|^{2} \quad n=1,2 \\
f_{\text {thru/line }}=\sqrt{\sum_{i=1}^{2} \sum_{j=1}^{2}\left|S_{i j}^{m}-S_{i j}^{s}\right|^{2}}
\end{gathered}
$$

\section{B. Calculation of PCB EM Equivalent Model}

Once the coaxial-to-waveguide transitions are characterized by the de-embedding technique, it is possible to obtain the PCB EM equivalent model by comparing the effects measured in the real enclosure loaded by the PCB and the ones computed in the virtual modeling.

The simulated scattering matrix of the whole metallic enclosure was computed as follows. The waveguide containing the PCB EM equivalent model was simulated with CST Microwave Studio which is based on the Finite Integration Technique and assuming a monomodal excitation. Afterwards, an analytical concatenation of the de-embedded transition 1, the waveguide containing the PCB equivalent model and the de-embedded transition 2 scattering matrices was computed by using Matlab functions that concatenate a cascade of scattering matrices. CST simulations were controlled by Matlab under Visual Basic Application (VBA) macro language, which provides an easy implementation of any optimization method.

The genetic algorithm and the gradient based technique were used in order to minimize the difference between simulated results and the measured data during the computation of the optimized PCB EM equivalent model. An individual from the genetic algorithm represents a possible solution of the problem that contains the estimation of the 3 different unknown parameters (thickness, dielectric constant and electrical conductivity) necessary to characterize the PCB EM equivalent model. The search limits for the optimization algorithms were: $[0.5,30] \mathrm{mm}$ for the dielectric thickness $(d)$, $[1,10]$ for the dielectric constant $\left(\varepsilon_{r}\right)$ and $[0,0.3] \mathrm{S} / \mathrm{m}$ for the electrical conductivity $(\sigma)$. Initially, a random population of 20 individuals was evaluated, while the crossover and mutation operations defined the following 20 generations evolving towards the optimum individual.

The fitting function for obtaining the proposed PCB EM equivalent model is 


$$
f_{p c b}=\frac{\sqrt{\sum_{i=1}^{2} \sum_{j=1}^{2} \sum_{k=1}^{N}\left|S_{i j k}^{\prime m}-S_{i j k}^{\prime s}\right|^{2}}}{N}
$$

where, $S_{i j k}^{\prime m}$ are the scattering parameters of the metallic enclosure containing the PCB measured with the vector network analyzer, $S_{i j k}^{\prime s}$ are the simulated scattering parameters of the whole enclosure containing the PCB EM model, $i$ and $j$ are the index reference port numbers for the scattering parameters, $k$ is the index for each frequency point and $N$ is the number of frequency points in the range under evaluation.

The frequency range to carry out the PCB parameters optimization process was restricted to those frequencies near the first measured resonance since this resonance was found to occur at the monomode range.

For the sweep algorithm the fitting function was evaluated with a set of $10 \times 10 \times 10$ sampled points equally spaced within the range of $[0.5,30] \mathrm{mm}$ for the thickness, $[0,0.3] \mathrm{S} / \mathrm{m}$ for the electrical conductivity and $[1,10]$ for the dielectric constant.

\section{EXPERIMENTAL SET UP}

The employed aluminium enclosure had a $30 \times 12 \times 30 \mathrm{~cm}^{3}$ volume, the same used in [4] and [5]. The testing probes were $4 \mathrm{~cm}$ long monopoles placed at $\pm 7.5 \mathrm{~cm}$ away from the top centre of the cavity as depicted in Fig. 4.

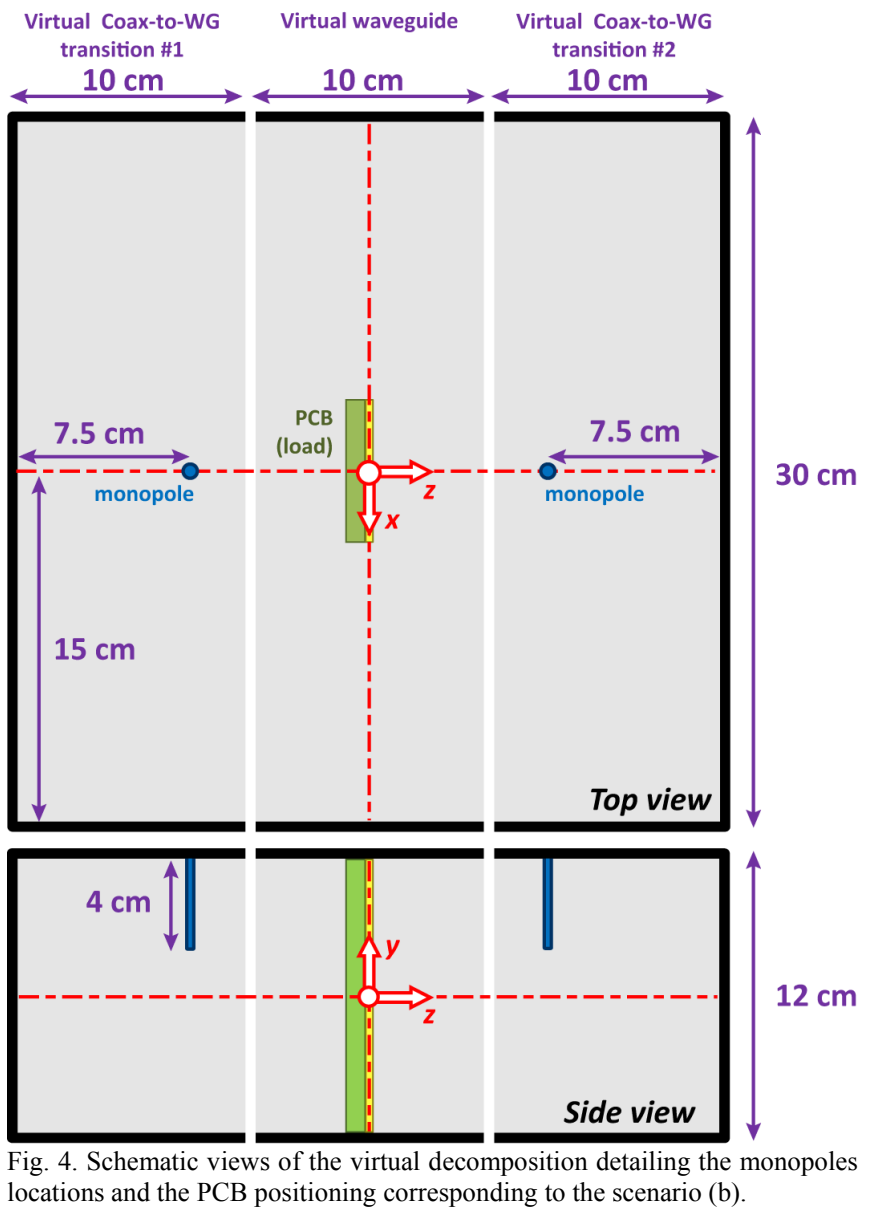

The analytic scattering matrix concatenation was implemented considering only monomode propagation. The first appearing mode for a $30 \times 12 \mathrm{~cm}^{2}$ waveguide cross section is the $\mathrm{TE}_{10}$ mode, which has a cut-off frequency of 500 $\mathrm{MHz}$. The $\mathrm{TE}_{20}$ is the following mode that could be propagated through the waveguide once its evanescent condition is overcame, that is, for frequencies above $1 \mathrm{GHz}$. Higher modes might appear starting from $1.249 \mathrm{GHz}$, which is the $\mathrm{TE}_{01}$.cut-off frequency Therefore, by placing the monopoles just in the minimum of the $\mathrm{TE}_{20}$ E-field pattern, which means an alignment to the $\mathrm{x}=0$ plane, it was ensured a wide monomode bandwidth up to $1.249 \mathrm{GHz}$.

A commercial PCB was placed inside the enclosure in order to obtain $S_{i j}^{\prime m}$ as a function of frequency. The commercial PCB was introduced at the central part of the metallic enclosure with three different representative orientations as depicted in Fig. 5. The recorded measurements were used together with the corresponding simulation results $S_{i j}^{\prime s}$ for computing the evaluation fitting function in (3).

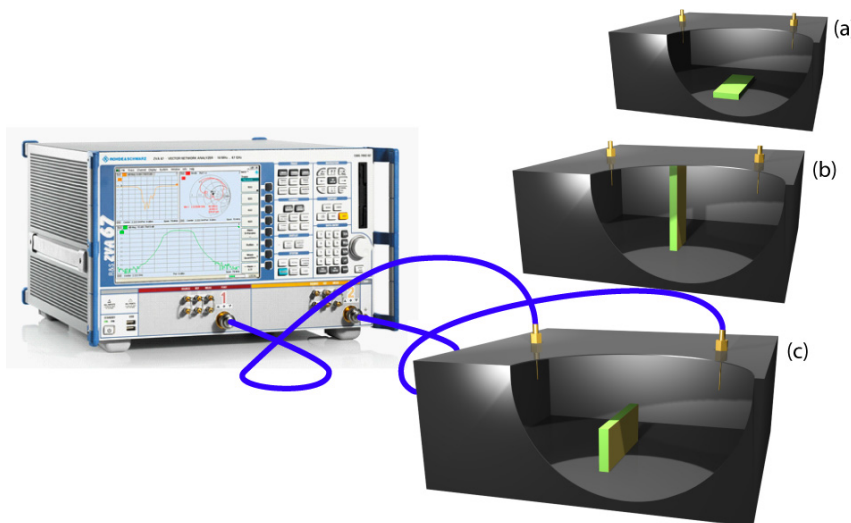

Fig. 5. Measurement setup for the different PCB configurations evaluated within the enclosure.

The commercial PCB dimensions were 12 x $6 \mathrm{~cm}^{2}$ approximately (Fig. 1). The PCB was double layered with grounding planes and tracks on both sides of the PCB while the electronic components were soldered to just one side. It has to be remarked that the PCB was lightly populated. The PCB was unpowered thus acting as a passive load. It was considered that the geometrical centre of the PEC layer of the EM equivalent model had to be aligned with the y-axis in the centre of the enclosure. The same consideration was taken in the experimental setups having the PCB board substrate as the alignment reference.

The scattering parameters were obtained by using a vector network analyser Rohde \& Schwarz ZVA67. The frequency range for the experimental study ranged from $500 \mathrm{MHz}$ to $1250 \mathrm{MHz}$. A frequency resolution of $1.97 \mathrm{MHz}$ was used during measurements.

\section{RESUlts}

Figs. 6 and 7 show the comparison of the $S_{21}$ magnitude and phase obtained for the transition 1 using the proposed inverse technique [6] and the three cavities method [10], respectively. 
As observed in both figures, both results agree very closely up to $1.25 \mathrm{GHz}$. Therefore, this comparison validates the calibration procedure for the coaxial-to-waveguide transitions. It must be clarified that the results have been obtained by assuming a monomode propagation through the virtual waveguide thru, but frequency axis is ranging beyond the cutoff frequency of the second propagating mode $\left(\mathrm{TE}_{20}\right)$. As it was explained above, this mode is not excited since the coaxial probes are aligned properly at the centre of the cavity as seen in Fig. 4, so $\mathrm{TE}_{2 \times \mathrm{m} 0}$ modes will not be excited. Similar results were obtained for transition 2 and the rest of scattering parameters of transition 1.

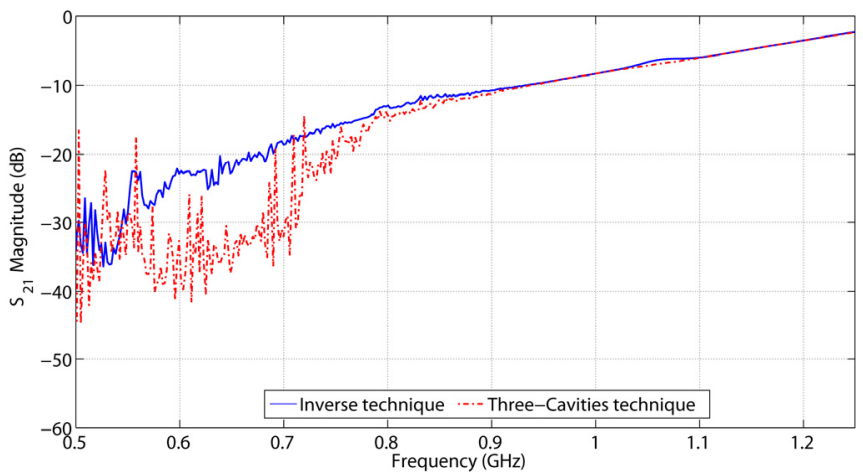

Fig. 6. Comparison of the $\left|S_{21}\right|$ obtained for the transition 1 using an inverse technique and the three cavities method.

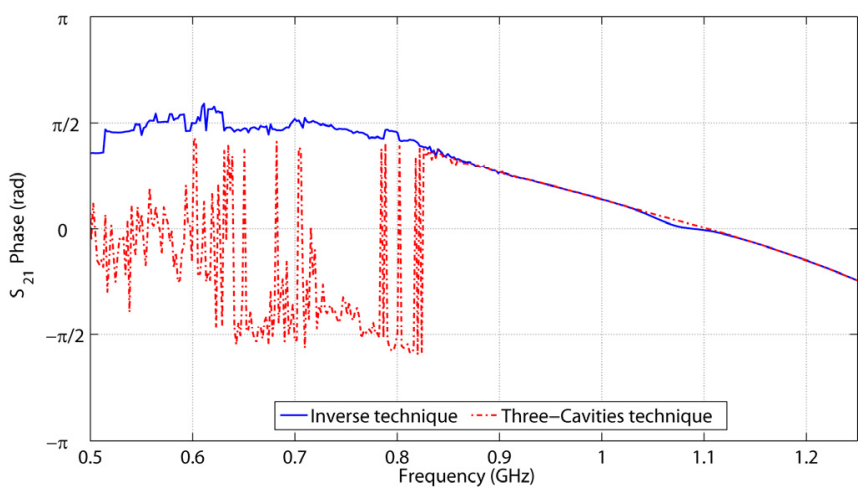

Fig. 7. Comparison of the phase of the $S_{21}$ obtained for the transition 1 using an inverse technique and the three cavities method.

The employed monopoles are short over the entire frequency range and especially ineffective at the lower frequencies. The low levels for the $\left|S_{21}\right|$ parameter at lower frequencies lead to slightly different values for both solutions as can be seen in Fig. 6. As it was expected, the inverse technique provides higher accuracy and precision than the three cavities method, because that calibration process includes an additional thru measurement. As a consequence, the differences at lower frequencies shown in Fig. 6 are also reproduced in Fig. 7. This is emphasized by the phase ambiguity due to the low values of $\left|S_{21}\right|$. Figs. 8 and 9 show the comparison of the $S_{21}$ magnitude and phase for the empty enclosure scenario and for three different cases: measurements, simulation results obtained by CST Microwave Studio and the results obtained by concatenating the scattering matrices of coaxial-to-waveguide transitions and the waveguide thru. From these data it can be concluded that there is a close matching between the proposed method and the measurements as expected, since the measurements of the empty enclosure were employed in the inverse technique calibration process in order to extract the scattering parameters of the transitions used in the calculations. Therefore, this also shows that the proposed calibration technique allows a precise and accurate characterization of the transitions.

Differences between CST simulations and measurements start at the resonance frequency values and increase gradually with frequency. This is due to the fact that simulations do not properly model the effect of monopoles coupling and therefore, any attempt of deriving an equivalent model based only in simulations would require a very highly detailed model. Calculated values at lower frequencies are noisy due to low $S_{21}$ transitions values at frequencies just above the cutoff frequency where the short monopoles are ineffective and the fundamental mode is starting to be excited.

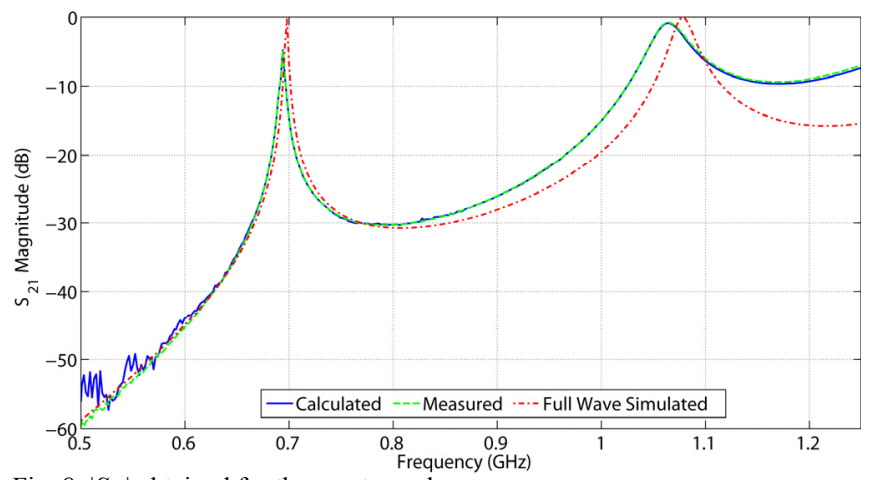

Fig. 8. $\left|S_{21}\right|$ obtained for the empty enclosure.

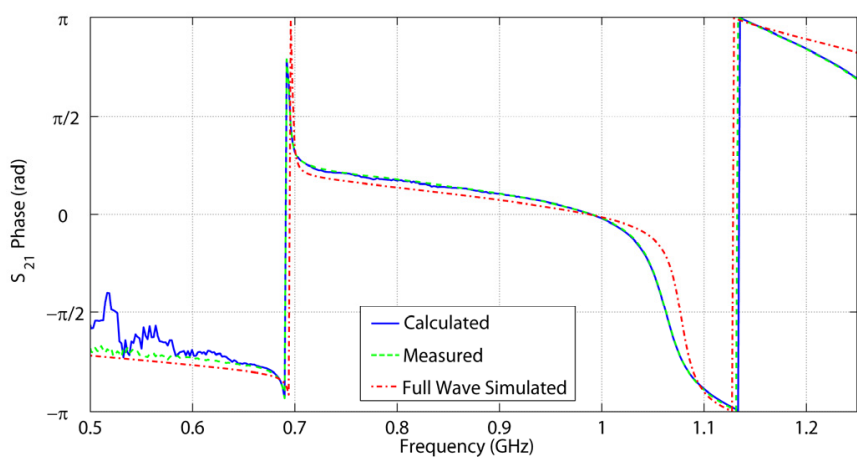

Fig. 9. Phase of $S_{21}$ obtained for the empty enclosure

Table I focuses on a comparison for the three traces shown in Fig. 8 and considers the quality factor, the resonance frequency and the maximum level for $\left|S_{21}\right|(\mathrm{dB})$ at the first resonance. The values have been extracted using a quadratic interpolation [11]. Consequently, accurate modelling of the empty enclosure using the inverse technique guarantees the precise extraction of the PCB EM equivalent model.

Three different approaches for evaluating the PCB EM equivalent model performance were carried out: a vast sweep of the three model parameters, the solution obtained by the genetic algorithm and a solution obtained by a gradient based optimization technique. Table II shows the best solutions obtained for each approach stated above, including the evaluation of the fitness function. For the scenarios (a) and (b) 
the frequency range under evaluation was limited to [650, 750] $\mathrm{MHz}$ and $[900,960] \mathrm{MHz}$ respectively, where the best monopole matching was found empirically under the monomode range (see Figs. 10 and 11). It can be concluded from these data that the three scenarios depicted in Fig. 5 produced different equivalents for the same PCB. In the case of scenario (c), despite the low values of the evaluation function the model was unable to reproduce the behaviour of the PCB inside the cabinet as it will be shown later.

TABLE I

EMPTY CAVITY PARAMETERS FOR THE FIRST RESONANCE

\begin{tabular}{lccc}
\hline \hline & $f_{\text {res }}(\mathrm{MHz})$ & $Q$ & $\left|\mathrm{~S}_{21}\right|(\mathrm{dB})$ \\
\hline Calculated & 694.03 & 210.669 & -4.724 \\
Measured & 694.03 & 210.326 & -4.710 \\
Simulated & 697.54 & 278.103 & -0.034
\end{tabular}

TABLE II

PCB EQUIVALENT MODELS FOR DIFFERENT SCENARIOS AND OPTIMIZATION TECHNIQUES

\begin{tabular}{clcccc}
\hline \hline \multirow{2}{*}{ Scenario } & $\begin{array}{c}\text { Optimization } \\
\text { Algorithm }\end{array}$ & $d(\mathrm{~mm})$ & $\varepsilon_{\mathrm{r}}^{\prime}$ & $\sigma(\mathrm{S} / \mathrm{m})$ & $f_{p c b}$ \\
& Sweep & 3.778 & 3.000 & 0.000 & 0.031 \\
(a) & GA & 2.911 & 4.914 & 0.024 & 0.031 \\
& Gradient & 3.804 & 2.748 & 0.023 & 0.033 \\
\hline \multirow{3}{*}{ (b) } & Sweep & 26.722 & 3.000 & 0.066 & 0.080 \\
& GA & 18.867 & 5.968 & 0.144 & 0.079 \\
& Gradient & 15.325 & 9.197 & 0.273 & 0.085 \\
\hline \multirow{3}{*}{ (c) } & Sweep & 30.000 & 1.000 & 0.033 & 0.085 \\
& GA & 7.1191 & 1.290 & 0.300 & 0.087 \\
& Gradient & 29.996 & 1.140 & 0.158 & 0.086
\end{tabular}

Fig. 10 shows the measurement data of $\left|S_{21}\right|$ when placing the PCB as depicted in scenario (a). Also in Fig. 10, the results obtained for the PCB equivalent model obtained by using GA and the simulations results obtained when simulating the whole structure with CST are depicted. It must be remarked that in this last case, CST used the same optimized values that the proposed technique to calculate the PCB model in order to observe the deviations of both approaches.

From the results, it can be concluded that the PCB model is capable of matching very closely the experimental data. On the contrary, when only using CST Microwave Studio software with the GA optimized data resonant frequencies and quality factors deviations are observed. Again those deviations are due to the effect of the monopole coupling. It must also be emphasized that in the scenario (a), the PCB position inside the cavity is not invasive enough to force a redistribution of the EM field energy within the enclosure. Consequently, resonances and levels for the $S_{21}$ curve vary slightly when compared to the empty cavity situation, so this case could be treated using a perturbational approach where the frequency deviation between the empty and the loaded cavity is directly related to the dielectric constant solution and the $\mathrm{Q}$ factor variation from the empty to the loaded cavity leads to the conductivity or loss tangent solution. The thickness influences both the resonant frequency and the $\mathrm{Q}$ factor. The opposite case is dealt with the scenario (b), where the PCB is placed in the middle of the enclosure where the most of the energy of the $\mathrm{TE}_{10}$ mode tends to concentrate when the enclosure is empty. However, besides the invasion of the PCB there is a vertical invariance due to its orientation.

Fig. 11 shows $\left|S_{21}\right|$ measured data, $\left|S_{21}\right|$ calculated by using the proposed technique and GA and the results obtained by simulating the whole structure with CST and the PCB optimized data for the scenario (b). From obtained data it can be derived that in this scenario the PCB model and the proposed technique are capable of reproducing the first resonance getting close to the second resonance. When using the PCB model with CST the results are poorer than for the previous case.

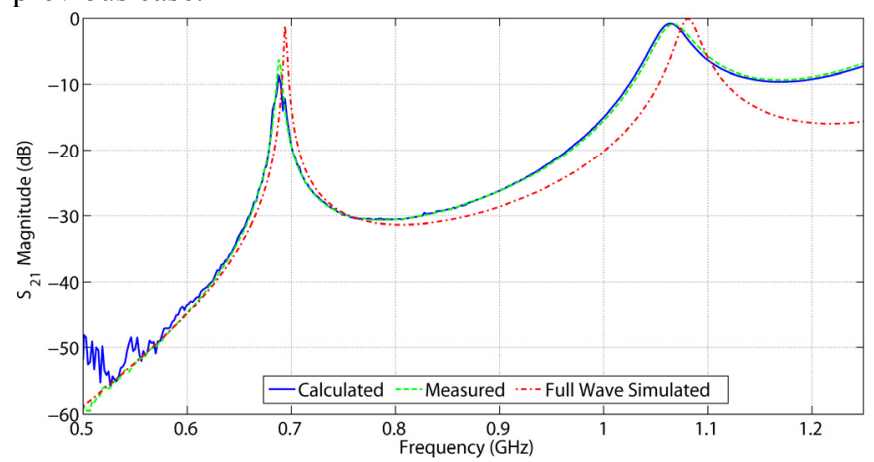

Fig. 10. $\left|S_{21}\right|$ obtained for the scenario (a) with a $d=2.911 \mathrm{~mm}, \varepsilon_{r}^{\prime}=4.914$ and $\sigma=0.024 \mathrm{~S} / \mathrm{m}$ for the PCB EM equivalent model versus CST simulations and measurements.

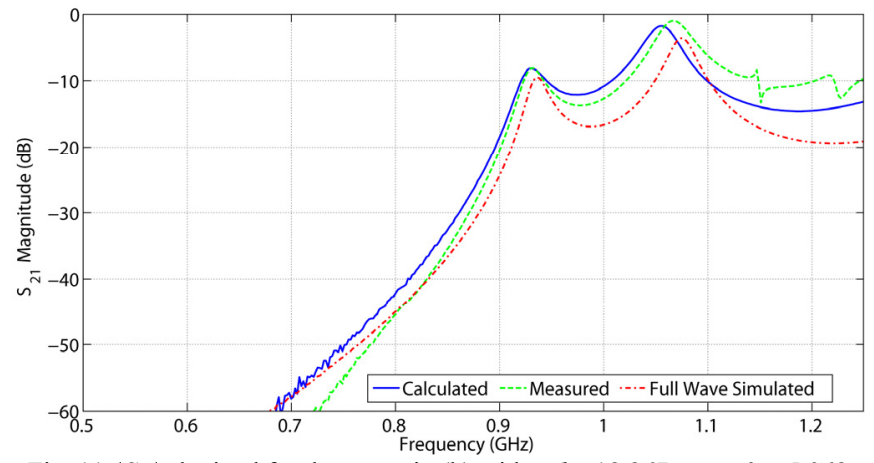

Fig. 11. $\left|S_{21}\right|$ obtained for the scenario (b) with a $d=18.867 \mathrm{~mm}, \varepsilon_{r}^{\prime}=5.968$ and $\sigma=0.144 \mathrm{~S} / \mathrm{m}$ for the PCB EM equivalent model versus CST simulations and measurements.

Fig. 12 shows the $\left|S_{21}\right|$ measured data, $\left|S_{21}\right|$ obtained with the GA PCB EM equivalent model and the CST simulation for the scenario (c). As in previous cases, CST simulations of the whole structure made use of the same optimized parameters than those used in the proposed technique. From obtained data it can be concluded that in this case the proposed method to extract the PCB EM equivalent model is not able to produce good results. This is due to the orientation of the PCB, which disrupts the vertical invariance along the y-axis becoming the most invasive scenario and producing higher order modes at the discontinuity that reach to the virtual ports of the central waveguide of Fig. 3. As it is known, $\mathrm{TE}_{10}$ mode exhibits vertical invariance, referred in this work as the $y$-axis. In the scenario (c) the PCB breaks up this condition as the PCB half fulfils the enclosure in height. To overcome this drawback it would be necessary to use a multimode calibration for both transitions and a multimode modelling for scenario (c). Since a monomode assumption has been used during all calculations 
the proposed technique is not able to produce good results for the first resonance.

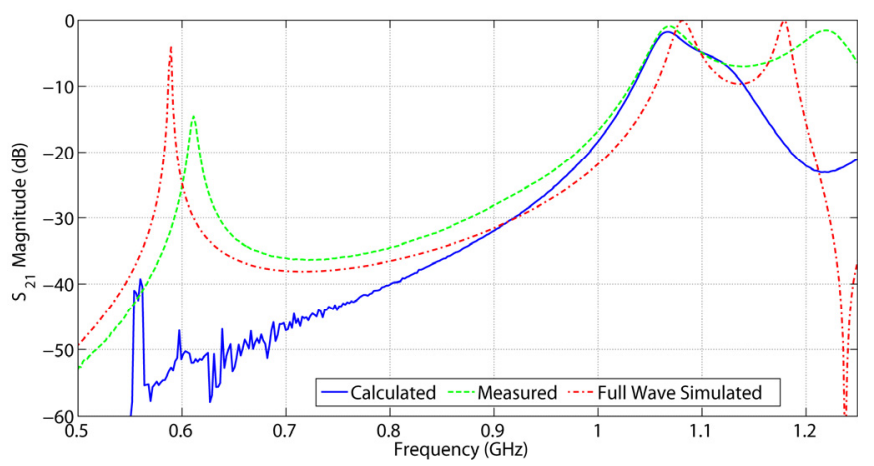

Fig. 12. $\left|S_{21}\right|$ obtained for the scenario (c) with a $d=7.119 \mathrm{~mm}, \varepsilon_{r}^{\prime}=1.290$ and $\sigma=0.300 \mathrm{~S} / \mathrm{m}$ for the PCB EM equivalent model versus CST simulations and measurements.

The values of the optimization evaluation function computed by using the sweep algorithm for the scenario (a) are presented in Figs. 13-15, where it is shown a 2D representation of $f_{p c b}$ by fixing one of the PCB model parameters to its corresponding closest optimized value obtained by the genetic algorithm shown in Table II. The same representations for the scenario (b) are shown in Figs. 16-18. The scale range for Figs. 13-18 has been fixed in order to appreciate the $f_{p c b}$ behaviour differences of scenarios (a) and (b).

The sweep results obtained for scenario (a) at Figs. 13 to 15 show that the fitting function for the error $\left(f_{p c b}\right)$ varies smoothly. This slow variation for scenario (a) can be explained by the fact that the PCB EM model is slightly perturbing the cavity electric fields. The stronger variation at these figures is shown in Fig. 14 where $f_{p c b}$ is shown versus thickness and dielectric constant. Also in these figures one can perceive that several minima can be found and, therefore, many PCB EM equivalent models are possible. As a consequence, global minimum search such as GA is important to find the best PCB model.

However, the behaviour of $f_{p c b}$ for scenario (b) is quite different as shown in Figs. 16 to 18. In this case, $f_{p c b}$ varies sharply with all variables. Only one area for minimum $f_{p c b}$ values is found at Figs. 16 and 18 where this function is depicted for different thickness-electrical conductivity and thickness-dielectric constant pairs.

From obtained results with scenario (b) at Fig. 17 it can be derived that several combinations of thickness and dielectric constant values may provide similar values for $f_{p c b}$ and consequently similar PCB EM equivalent models, at least to match the first resonance. This can be explained by the fact that the combination of both variables provides similar electrical volumes within the resonant cavity and, therefore, these combinations provide similar resonant frequencies.

Again in the case of scenario (b), results indicate that it is important to use global optimization techniques to find the parameters of the optimized PCB EM model.

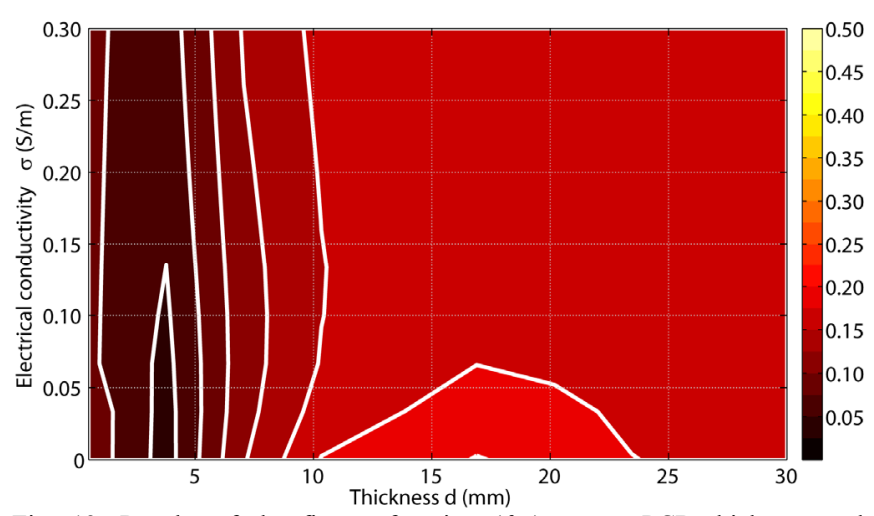

Fig. 13. Results of the fitness function $\left(f_{p c b}\right)$ versus PCB thickness and electrical conductivity for scenario (a). $\varepsilon_{r}=5$.

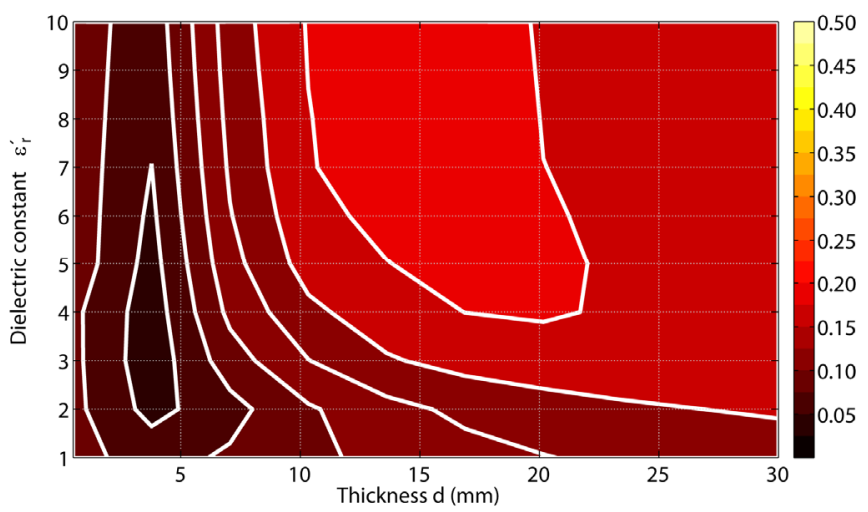

Fig. 14. Results of the fitness function $\left(f_{p c b}\right)$ versus PCB thickness and dielectric constant for scenario (a). $\sigma=0.033 \mathrm{~S} / \mathrm{m}$.

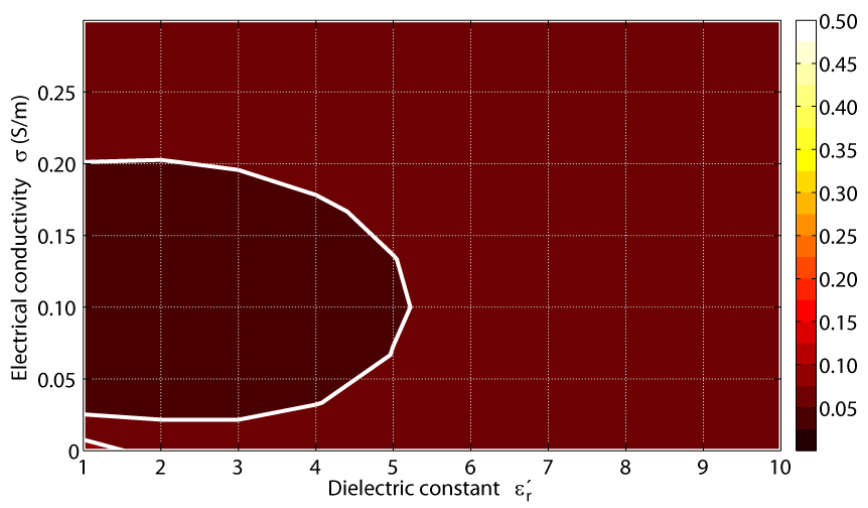

Fig. 15. Results of the fitness function $\left(f_{p c b}\right)$ versus PCB dielectric constant and electrical conductivity for scenario (a). $d=3.778 \mathrm{~mm}$.

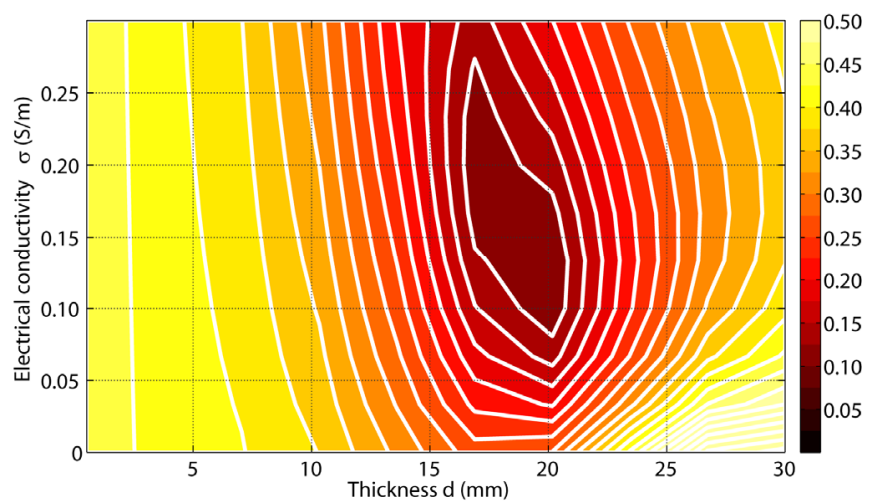

Fig. 16. Results of the fitness function $\left(f_{p c b}\right)$ versus PCB thickness and electrical conductivity for scenario (b). $\varepsilon_{r}{ }^{\prime}=6$. 


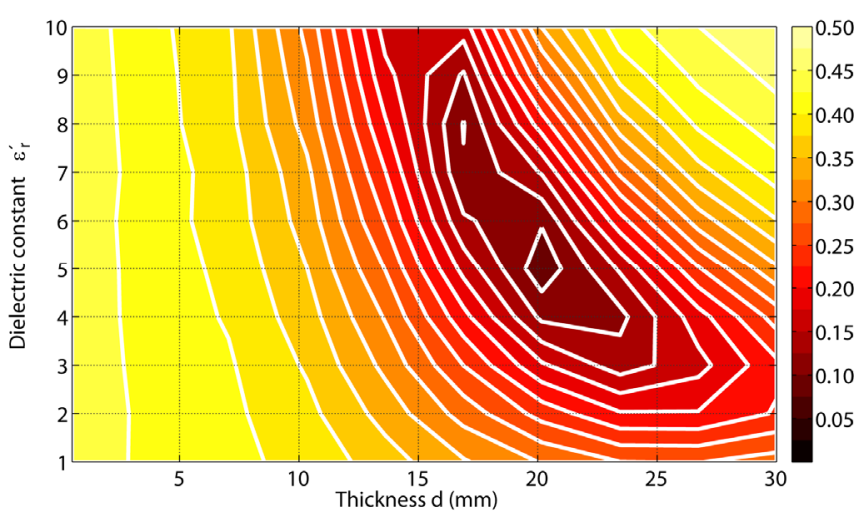

Fig. 17. Results of the fitness function $\left(f_{p c b}\right)$ versus PCB thickness and dielectric constant for scenario (b). $\sigma=0.133 \mathrm{~S} / \mathrm{m}$.

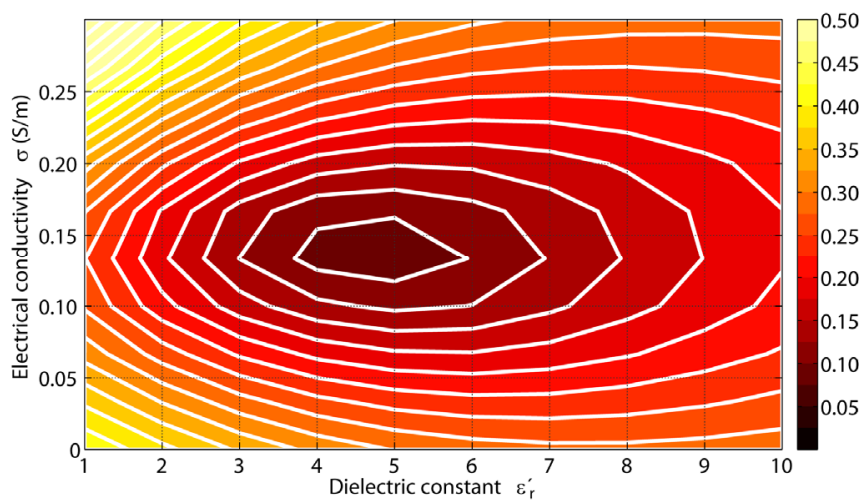

Fig. 18. Results of the fitness function $\left(f_{p c b}\right)$ versus PCB dielectric constant and electrical conductivity for scenario (b). $d=20.167 \mathrm{~mm}$.

Finally, computation times obtained using CST Microwave Studio simulation tool lead to 31 seconds in the case of the thru for the proposed technique in scenario (b) and 130 seconds for the whole enclosure set up. An Intel Core 2 Quad (a) $2.66 \mathrm{GHz}$ with $4 \mathrm{~GB}$ RAM was used during the simulations. Time benefits derived from the reduction in the computational domain in optimization processes are clearly justified.

\section{V.CONCLUSION}

In this work, a new method for extracting PCB EM equivalent models within metallic enclosures has been proposed and validated. The method consists of two different stages. During the first step the whole metallic enclosure is virtually divided into three different parts (a waveguide thru and two coaxial-towaveguide transitions). The coaxial-to-waveguide transitions used at the experimental set up are precisely characterized by means of an inverse procedure that provides their complex scattering matrices. Three sliding shorts and a waveguide thru are used in this work to carry out this experimental procedure. Next, during the second step the experimental scattering matrices of the coaxial-to-waveguide transitions and the results of CST Microwave Studio simulations modeling a waveguide thru that contains the PCB EM model are concatenated and compared to experimental measurements in order to obtain the three parameters of the equivalent model: thickness, dielectric constant and electrical conductivity.

Results show that this method increases the accuracy of the PCB EM equivalent model versus a model extracted only with simulations because the effect of coaxial probes is not transferred to the PCB model. Simulations or analytical models may obtain very good results, but tolerances in the manufacturing of the device under test due to mechanization processes or soldering may lead to inaccuracies in their results.

Several combinations of thickness, dielectric constant and electrical conductivity values can provide very similar values for the equivalent model fitting. This is due to the fact that the dielectric constant and the dielectric volume of the PCB model establish the resonance frequency within the cavity whereas the electric conductivity and dielectric volume control resonance bandwidth. A strong correlation between the dielectric constant and the thickness of the model can also be observed.

Precise PCB EM equivalent models have been obtained for scenarios (a) and (b) where the perturbation introduced by PCB orientation did not generate an intermodal energy scattering. It must be remarked that different PCB EM equivalent models were obtained for several PCB configurations within the enclosure. This means that the model is not an approximate geometrical model, but an accurate EM model considering the interactions with the enclosure.

However, for scenario (c) the proposed method failed due to the fact that the main considered hypothesis was monomode propagation at the virtual waveguide thru ( $\mathrm{TE}_{10}$ in this case). A multimode version of the proposed techniques would be needed to reach a solution in this case. More work is envisaged in this direction.

Computation time reduction is also a key benefit derived from the proposed technique.

\section{REFERENCES}

[1] R. Azaro, S. Caorsi, M. Cosso, G. M. Costini, M. Donelli, R. Ene, G. L. Gragnani, and M. Pastorino, "A semianalytical approach for the evaluation of radiated immunity on a printed-circuit board in metallic enclosures", Microwave Opt. Technol. Lett., 27, pp. 204-207, November 2000 .

[2] W. Wallyn, and D. De Zutter, "Modeling the shielding effectiveness and resonances of metallic shielding enclosures loaded with PCBs", IEEE International Symposium on Electromagnetic Compatibility, vol. 2, pp. 691-696, August 2001.

[3] M. P. Robinson, S.J. Porter and P Op gen Oorth, "Reflection and transmission coefficients of printed circuit boards", $4^{\text {th }}$ Eur. Symp. EMC Brugge, September 2000.

[4] D. W. P. Thomas, A. C. Denton, T. Konefal, T. Benson, C. Christopoulos, J. F. Dawson, A. Marvin, and S. J. Porter, "Characterisation of the shielding effectiveness of loaded equipment enclosures", International Conference and Exhibition on Electromagnetic Compatibility, pp. 89-94. July 1999.

[5] D. W. P. Thomas, A. C. Denton, T. Konefal, T. Benson, C. Christopoulos, J. F. Dawson, A. Marvin, S. J. Porter, and P. Sewell, "Model of the electromagnetic fields inside a cuboidal enclosure populated with conducting planes o printed circuit boards", IEEE Trans. on Electromagn. Compat., vol.43, no. 2, pp. 161-169, May 2001.

[6] A. J. Lozano-Guerrero, F. J. Clemente-Fernández, J. Monzó-Cabrera, J. L. Pedreño-Molina, A. Díaz-Morcillo,"Precise evaluation of coaxial to waveguide transitions by means of inverse techniques", IEEE Trans. on Microw.Thoeory Tech., vol.58, no. 1, pp. 229-235, January 2010.

[7] T. Konefal, J. F. Dawson, A. Denton, T. M. Benson, C. Christopoulos, A. C. Marvin, S. J. Porter and D. W. P. Thomas, "Electromagnetic field predictions inside screened enclosures containing radiators", in Proc. Inst. Elect. Eng. Conf. Electromagnetic Compatibility, vol. 464, pp. 95100, July 1999. 
[8] A. Lozano, M. P. Robinson, A. Diaz-Morcillo, and J. V. Balbastre, "Evaluation and optimization of an equivalent model for printed circuit boards inside metallic enclosures", General Assembly URSI, Chicago, USA, August 2008

[9] A. C. Marvin, J. F. Dawson, S. Ward, L. Dawson, J. Clegg, and A. Weisenfeld, "A proposed new definition and measurement of the shielding effect of equipment enclosures", IEEE Trans. on Electromagn. Compat., vol.46, no. 3, pp. 459-468, August 2004.

[10] J. F. Liang, H. Chang and K. A. Zaki, "Coaxial probe modeling in waveguides and cavities," IEEE Trans. Microw. Theory Tech., vol.40, no.12, pp.2172-2180, December 1992.

[11] M. P. Robinson and J. Clegg, "Improved determination of Q-factor and resonant frequency by a quadratic curve-fitting method", IEEE Trans. on Electromagn. Compat., vol.47, no. 2, pp. 399-402, May 2005.

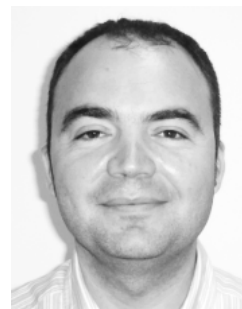

Antonio José Lozano-Guerrero was born in E Verger, Spain, in 1976. He received the Dipl. Ing. degree in Telecommunications engineering from the Universidad Politécnica de Valencia (UPV), Valencia, Spain in 2003 and his Ph.D. degree at the Universidad Politécnica de Cartagena (UPCT), Cartagena, Spain in 2008. From 2003 to 2004, he was a Research Assistant with the Department of Communications, UPV. In 2004, he joined the Department of Information Technologies and Communications, UPCT, where he is currently an Associate Lecturer. His current research areas are Electromagnetic Compatibility, Numerical Techniques in Electromagnetism and Industrial Microwave Heating Systems.

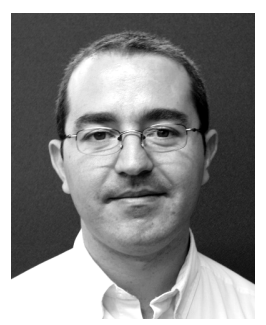

Juan Monzó-Cabrera was born in Elda (Alicante), Spain, on January 1973. He received the Dipl. Ing. and Ph.D. degrees in Telecommunications engineering from the Universidad Politécnica de Valencia. He works nowadays as an Associate Lecturer at Universidad Politécnica de Cartagena. He has co-authored more than 60 papers in referred journals and conference proceedings and holds several patents regarding microwave heating processes. Dr. Monzó-Cabrera is the General Secretary of the Association of Microwave Power in Europe for Research and Education (AMPERE), a European association devoted to RF and microwave energy promotion. His current research areas cover microwave-assisted heating and numerical techniques in electromagnetism.

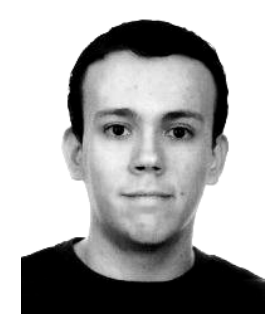

Francisco Javier Clemente-Fernández was born in La Unión (Murcia), Spain, in 1985. He received in 2008 the Dipl. Ing. degree in telecommunications engineering from the Universidad Politécnica de Cartagena (UPCT), Cartagena, Spain. In 2008, he joined the Department of Information Technologies and Communications, UPCT, where he is currently working toward the Ph.D. degree. His main research areas are microwave-assisted heating and drying processes and microwave filters design and optimization.

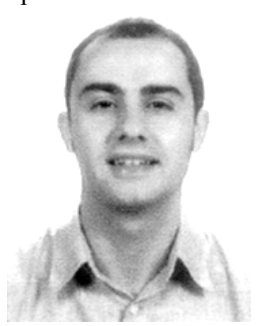

José Fayos-Fernández was born in Valencia, Spain, in September 1976. He received the Dipl. Ing. degree in Telecommunications Engineering from the Universidad Politécnica de Valencia (UPV) in 2001. He was with the ITACA Institute, UPV, working on the radiation level certification campaign of radioelectric stations in 2002. From October 2002 to early 2004, he was a Research Assistant with the Universidad Politécnica de Cartagena (UPCT), Cartagena, Spain, where he focused his research on electromagnetic dosimetry. He was an academic guest at Swiss Federal Institute of Technology in Zurich (ETHZ) for 6 months since September 2006, researching on EM exposure setups for electromagnetic dosimetry in vitro studies with the IT'IS Foundation. He received his Ph.D degree from UPCT in 2009. Since 2004 he has been a lecturer with the School of Telecommunication Engineering, UPCT. In 2010 and 2011 he was awarded as a reference lecturer in the European Space of Higher Education by the UPCT.

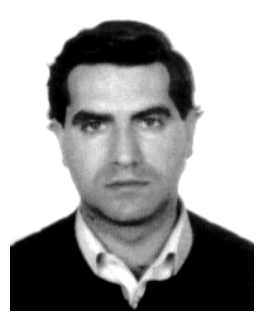

Juan L. Pedreño-Molina was born in Cartagena (Murcia), 1969. He received in 1984 his BASc. from the Universidad Politécnica de Madrid, Spain (UPM) and the Ph.D. in Neurotechnology, Control and Robotics in 2000. He is Assistant Professor of Telecommunication Engineering at the Universidad Politécnica de Cartagena (UPCT), SPAIN. Since 1999 he has belonged to the Department of Information Technologies and Communications at UPCT. His research interests are in Signal Processing applied to Optimization and Control of Nonlinear Systems, and Modeling based on Neural Networks with applications to robotics and drying processes.

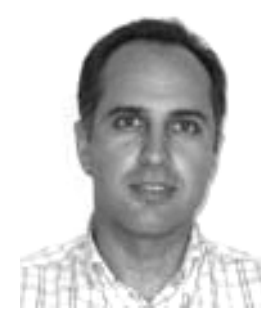

Alejandro Díaz-Morcillo (S'95-M'02-SM'09) received the Ingeniero (Ms. Eng.) and Doctor Ingeniero ( $\mathrm{Ph}$. D.) degrees in Telecommunication Engineering, both from Universidad Politécnica de Valencia (UPV), Spain, in 1995 and 2000, respectively. From 1996 to 1999 he was a Research Assistant at the Department of Communications of the UPV, and in 1999 he joined the Department of Information Technologies and Communications at the Universidad Politécnica de Cartagena (UPCT), Spain, as Teaching Assistant, where he is currently Professor. He leads the "Electromagnetics and Matter" Research Group at UPCT and his main research interest lays on Numerical Methods in Electromagnetics, Industrial Microwave Heating Systems and Dielectric Characterization. 